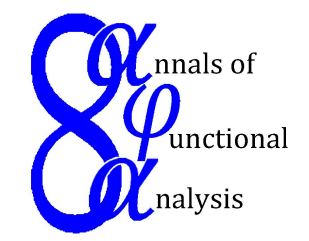

Ann. Funct. Anal. 6 (2015), no. 3, 29-41

http://doi.org/10.15352/afa/06-3-3

ISSN: 2008-8752 (electronic)

http://projecteuclid.org/afa

\title{
ASCOLI-TYPE THEOREMS FOR LOCALLY BOUNDED QUASICONTINUOUS FUNCTIONS, MINIMAL USCO AND MINIMAL CUSCO MAPS
}

\author{
DUŠAN HOLÝ \\ Communicated by V. Vesko
}

\begin{abstract}
Let $X$ be a locally compact space and let $(Y, d)$ be a nontrivial metric space such that $d$ has the Heine-Borel property. We prove Ascoli-type theorem for locally bounded quasicontinuous functions from $X$ to $Y$. Using the above result we also prove an Ascoli-type theorems for the spaces of minimal usco and minimal cusco maps from $X$ to $\mathbb{R}$.
\end{abstract}

\section{INTRODUCTION}

In our paper we prove Ascoli-type theorems for locally bounded quasicontinuous functions, minimal usco and minimal cusco maps.

The notion of quasicontinuity of real-valued functions of real variable was introduced by Kempisty in [16]. The property of quasicontinuity was pehaps the first time used by Baire in [1] in the study of points of continuity of separately continuous functions. For general topological spaces $X$ and $Y$ a function $f: X \rightarrow Y$ is quasicontinuous [19] at $x \in X$ if for every open set $V \subset Y, f(x) \in V$ and open set $U \subset X, x \in U$ there is a nonempty open set $W \subset U$ such that $f(W) \subset V$. If $f$ is quasicontinuous at every point of $X$, we say that $f$ is quasicontinuous.

Quasicontinuous functions are also very important in the study of minimal usco and minimal cusco maps. In fact, every selection of of a minimal usco map is quasicontinuous.

There is a rich literature concerning the study of quasicontinuity (see, for instance [19], [17], [4]).

Date: Received: Jul. 1, 2014; Accepted: Oct. 29, 2014.

2010 Mathematics Subject Classification. Primary 46B25; Secondary 54C60.

Key words and phrases. Banach space, quasicontinuous function, densely equiquasicontinuous, minimal usco map, minimal cusco map. 
The acronym usco (cusco) stands for a (convex) upper-semicontinuous nonempty compact-valued set-valued map. Such set-valued maps are interesting because they describe common features of maximal monotone operators, of the convex subdifferential and of Clarke generalized gradient. (see [5])

Minimal usco and minimal cusco maps are used in many papers (see [5], [7], [13], [10], [11], [12]). Minimal usco maps are a very convenient tool in the theory of games (see [6]) or in functional analysis. (see [3]).

\section{Compact subsets of $\left(Q^{*}(X, Y), \tau_{U C}\right)$}

Let $X, Y$ be Hausdorff topological spaces. By $\mathbb{N}$ we denote the set of positive integers, $\mathbb{R}$ be the space of real numbers with the usual Euclidean metric and $\mathbb{R}^{n}$ be the finite dimensional Euclidean space. The symbol $\bar{A}$ and $\operatorname{Int} A$ will stand for the closure and interior of the set $A$ in a topological space.

We say that a subset of $X$ is quasi-open (or semi-open) if it is contained in the closure of its interior. Then a function $f: X \rightarrow Y$ is quasicontinuous if and only if $f^{-1}(V)$ is quasi-open for every open set $V \subset Y$.

Denote by $F(X, Y)$ the set of all functions from $X$ to $Y$, by $C(X, Y)$ the set of all continuous functions in $F(X, Y)$, and by $Q(X, Y)$ the set of all quasicontinuous functions from $X$ to $Y$. Notice that if $f: X \rightarrow Y$ is a function, we will use the symbol $f$ also for the graph of $f$.

By $\tau_{p}$ and $\tau_{U C}$ we mean the topology of pointwise convergence on $F(X, Y)$ and the topology of uniform convergence on compact sets on $F(X, Y)$, respectively.

Let $H \subset F(X, Y)$ and let $x \in X$, denote by $H[x]$ the set $\{f(x) \in Y ; f \in H\}$.

If $X$ is a locally compact space and $(Y, d)$ is a metric space, the Ascoli theorem [15] says that a subset $\mathcal{E}$ of $\left(C(X, Y), \tau_{U C}\right)$ is compact if and only if it is closed in $\left(C(X, Y), \tau_{U C}\right), \mathcal{E}[x]$ has a compact closure for each $x \in X$ and is equicontinuous, where a subset $\mathcal{E}$ of $C(X, Y)$ is equicontinuous provided that for each $x \in X$ and $\epsilon>0$ there is a neighbourhood $U$ of $x$ with $d(f(x), f(z))<\epsilon$ for all $z \in U$ and $f \in \mathcal{E}$.

If $f$ is a function from $X$ to a metric space $(Y, d)$, we say that $f$ is locally bounded, if for every $x \in X$ there is an open set $U \subset X, x \in U$ such that $f(U)=\{f(u): u \in U\}$ is a bounded subset of $(Y, d)$. Denote by $F^{*}(X, Y)$ the space of all locally bounded functions from $F(X, Y)$. If $G$ is a subset of $F^{*}(X, Y)$ then we denote this set also by $G^{*}$.

Inspired by papers [9], [18] we give the following definition.

Definition 2.1. Let $X$ be a topological space and $(Y, d)$ be a metric space. We say that a subset $\mathcal{E}$ of $F^{*}(X, Y)$ is densely equiquasicontinuous at a point $x$ of $X$ provided that for every $\epsilon>0$, there exists a finite family $\mathcal{B}$ of subsets of $X$ which are either quasi-open or nowhere dense such that $\cup \mathcal{B}$ is a neighbourhood of $x$ and such that for every $f \in \mathcal{E}$, for every $B \in \mathcal{B}$ and for every $p, q \in B$, $d(f(p), f(q))<\epsilon$. Then $\mathcal{E}$ is densely equiquasicontinuous provided that it is densely equiquasicontinuous at every point of $X$. 
Lemma 2.2. Let $X$ be a topological space and $(Y, d)$ be a metric space. If $\mathcal{E}$ is densely equiquasicontinuous subset of $C(X, Y)$, then $\mathcal{E}$ is equicontinuous.

Proof. Let $x \in X$ and $\epsilon>0$. Because $\mathcal{E}$ is densely equiquasicontinuous, there exist a finite family $\mathcal{B}=\left\{B_{1}, B_{2}, \cdots, B_{n}\right\}$ of quasi-open or nowhere dense subsets of $X$ such that $\cup \mathcal{B}$ is a neighborhood of $x$ and such that for every $f \in \mathcal{E}$, for every $B \in \mathcal{B}$ and for every $p, q \in B, d(f(p), f(q))<\frac{\epsilon}{2}$. Since $\mathcal{E} \subset C(X, Y)$, for every $f \in \mathcal{E}$, for every $B \in \mathcal{B}$ and for every $p, q \in \bar{B}, d(f(p), f(q))<\epsilon$. Without loss of generality we can suppose that $x \in \overline{B_{i}}$ for every $i \in\{1,2, \cdots, n\}$. Now let $z \in \cup \mathcal{B}$ and $f \in \mathcal{E}$. Then there is $i \in\{1,2, \cdots, n\}$ such that $z \in B_{i}$ and so $d(f(x), f(z))<\epsilon$.

Remark 2.3. Note that if $\left\{A_{1}, A_{2}, \cdots, A_{n}\right\}$ is a family of quasi-open sets of $X$, then the set $A=A_{1} \cap \cdots \cap A_{n}$ is nowhere dense or it is a union of nowhere dense set and quasi-open set. In fact, consider first the case $\operatorname{Int} A=\emptyset$. If $x \in A$, then there is $i \in\{1,2, \cdots, n\}$ such that $x \in A_{i} \backslash \operatorname{Int} A_{i}$, hence $A \subset$ $A_{1} \backslash$ Int $A_{1} \cup \cdots \cup A_{n} \backslash \operatorname{Int} A_{n}$. Since $A_{i}$ is quasi-open for every $i \in\{1,2, \cdots, n\}$, $A$ is nowhere dense. Now let $\operatorname{Int} A \neq \emptyset$. Then we can show as above that the set $A \backslash \operatorname{Int} A$ is nowhere dense and so $A=(A \backslash \operatorname{Int} A) \cup \operatorname{Int} A$ is a union of a nowhere dense set $A \backslash \operatorname{Int} A$ and a quasi-open set $\operatorname{Int} A$.

Lemma 2.4. Let $X$ be a topological space and $(Y, d)$ be a metric space such that every bounded set is totally bounded. If $\mathcal{E}$ is a densely equiquasicontinuous subset of $F^{*}(X, Y)$ and $f \in Q^{*}(X, Y)$, then $\mathcal{E} \cup\{f\}$ is densely equiquasicontinuous.

Proof. Let $x \in X$ and let $\epsilon>0$. Since $\mathcal{E}$ is densely equiquasicontinuous at $x$ there exists a finite family $\mathcal{B}=\left\{B_{1}, B_{2}, \cdots, B_{n}\right\}$ of subsets of $X$ which are either quasi-open or nowhere dense such that $\cup \mathcal{B}$ is a neighbourhood of $x$ and such that for every $f \in \mathcal{E}$, for every $B \in \mathcal{B}$ and for every $p, q \in B, d(f(p), f(q))<\epsilon$. Let $O$ be a neighbourhood of $x$ such that $O \subset \cup \mathcal{B}$ and $f(O)$ is bounded. Choose $y_{0} \in Y$ and let $r>0$ be such that the set $f(O)$ is contained in the closed ball $B_{r}\left(y_{0}\right)$. Let $V_{1}, V_{2}, \cdots, V_{m}$ be a finite open cover of $B_{r}\left(y_{0}\right)$ where radius of $V_{j}$ is less than $\epsilon$ for every $j \in\{1, \cdots, m\}$. For every $j \in\{1,2, \cdots, m\}$ let $H_{j}=f^{-1}\left(V_{j}\right)$. Then $\mathcal{H}=\left\{H_{j}: j \in\{1,2, \cdots, m\}\right\}$ is a finite family of quasi-open subsets of $X$ such that $O \subset \cup \mathcal{H}$. For every $i \in\{1,2, \cdots, n\}, j \in\{1,2, \cdots, m\}$ put $P_{i, j}=\left(B_{i} \cap H_{j}\right) \backslash \operatorname{Int}\left(B_{i} \cap H_{j}\right)$ and $R_{i, j}=\operatorname{Int}\left(B_{i} \cap H_{j}\right)$, where $P_{i, j}$ is a nowhere dense set (if $B_{i}$ and $H_{j}$ are both quasi-open see Remark 2.3).

Denote by $\mathcal{D}$ the finite family containing all nonempty sets $P_{i, j}$ and $R_{i, j}$ where $i \in\{1,2, \cdots, n\}, j \in\{1,2, \cdots, m\}$. Evidently $O \subset \cup \mathcal{D}$ and so $\cup \mathcal{D}$ is a neighbourhood of $x$. It is easy to see that for every $g \in \mathcal{E} \cup\{f\}$, for every $D \in \mathcal{D}$ and for every $p, q \in D, d(g(p), g(q))<\epsilon$.

It should be noted that bounded sets are totally bounded for a metric $d$ if and only if the metric of its completion has the Heine-Borel property.

Lemma 2.5. Let $X$ be a topological space and $(Y, d)$ be a metric space. Let $\left\{f_{\lambda}: \lambda \in \Lambda\right\}$ be a net in $Q^{*}(X, Y)$ pointwise convergent to $f \in F(X, Y)$ and let the set $\left\{f_{\lambda}: \lambda \in \Lambda\right\}$ be densely equiquasicontinuous. Then $f \in Q^{*}(X, Y)$. 
Proof. Let $x \in X$. Suppose, by the way of contradiction, that $f$ is not quasicontinuous at $x$. Then there exist $\epsilon>0$ and an open set $U, x \in U$ such that for every nonempty open set $W \subset U$ there is $z \in W$ such that $d(f(x), f(z))>\epsilon$.

Since $\left\{f_{\lambda}: \lambda \in \Lambda\right\}$ is densely equiquasicontinuous at $x$, there exists a finite family $\mathcal{B}=\left\{B_{1}, B_{2}, \cdots, B_{n}\right\}$ of subsets of $X$ which are either quasi-open or nowhere dense such that $\cup \mathcal{B}$ is a neighbourhood of $x$ and such that for every $\lambda \in \Lambda$, for every $B \in \mathcal{B}$ and for every $p, q \in B, d\left(f_{\lambda}(p), f_{\lambda}(q)\right)<\frac{\epsilon}{4}$. Without loss of generality we can suppose that $x \in \overline{B_{i}}$ for every $i \in\{1,2, \cdots, n\}$.

Denote by $C$ the set of all numbers from $j \in\{1,2, \cdots, n\}$ where $\operatorname{Int} B_{j} \neq \emptyset$. We choose a $z_{j} \in \operatorname{Int} B_{j} \cap U$ such that $d\left(f(x), f\left(z_{j}\right)\right)>\epsilon$ for every $j \in C$. Since the net $\left\{f_{\lambda}: \quad \lambda \in \Lambda\right\}$ is pointwise convergent to $f$, there is $\lambda \in \Lambda$ such that $d\left(f(x), f_{\lambda}(x)\right)<\frac{\epsilon}{4}$ and $d\left(f\left(z_{j}\right), f_{\lambda}\left(z_{j}\right)\right)<\frac{\epsilon}{4}$ for every $j \in C$.

We show that for $\frac{\epsilon}{4}$, the open set $U \cap($ Int $\cup \mathcal{B})$ and for every nonempty open set $W \subset(U \cap(I n t \cup \mathcal{B}))$ there exists $w \in W$ such that $d\left(f_{\lambda}(x), f_{\lambda}(w)\right)>\frac{\epsilon}{4}$. So let $W \subset(U \cap(\operatorname{Int} \cup \mathcal{B}))$. Let $j \in C$ be such that $\operatorname{Int} B_{j} \cap W \neq \emptyset$ and let $w \in \operatorname{Int} B_{j} \cap W$. Then $d\left(f_{\lambda}(w), f_{\lambda}\left(z_{j}\right)\right)<\frac{\epsilon}{4}$. We have

$$
d\left(f_{\lambda}(w), f\left(z_{j}\right)\right) \leq d\left(f_{\lambda}(w), f_{\lambda}\left(z_{j}\right)\right)+d\left(f_{\lambda}\left(z_{j}\right), f\left(z_{j}\right)\right)<\frac{\epsilon}{4}+\frac{\epsilon}{4}=\frac{2 \epsilon}{4}
$$

and $d\left(f_{\lambda}(x), f(x)\right)<\frac{\epsilon}{4}$. So from inequality $d\left(f(x), f\left(z_{j}\right)\right)>\epsilon$ follows that $d\left(f_{\lambda}(x), f_{\lambda}(w)\right)>\frac{\epsilon}{4}$. Hence $f_{\lambda}$ is not quasicontinuous at $x$, a contradiction.

Suppose, by the way of contradiction, that $f$ is not locally bounded at $x$. Choose any $y_{0} \in Y$. Let $U$ be a neighbourhood of $x$. For every $n \in \mathbb{N}$ there is $x_{n} \in U \cap \operatorname{Int} \cup \mathcal{B}$ such that $d\left(f\left(x_{n}\right), y_{0}\right)>n$. By passing to a subsequence there is $B_{i} \in \mathcal{B}$ such that $\left\{x_{n}: n \in \mathbb{N}\right\} \subset B_{i}$. The net $\left\{f_{\lambda}: \lambda \in \Lambda\right\}$ pointwise converges to $f$, so for every $n \in \mathbb{N}$ there is $\lambda_{n} \in \Lambda$ such that $d\left(f_{\lambda}\left(x_{n}\right), y_{0}\right)>n$ for every $\lambda>\lambda_{n}$. Then, since the set $\left\{f_{\lambda}: \lambda \in \Lambda\right\}$ is densely equiquasicontinuous at $x$, $d\left(f_{\lambda}(z), y_{0}\right)>n-\frac{\epsilon}{4}$ for every $z \in B_{i}$ and for every $\lambda>\lambda_{n}$. From this it follows that $d\left(f(z), y_{0}\right) \geq n-\frac{\epsilon}{4}$ for every $n \in \mathbb{N}$ and for every $z \in B_{i}$, a contradiction.

Corollary 2.6. Let $X$ be a topological space and $(Y, d)$ be a metric space. Let $\mathcal{E}$ be a densely equiquasicontinuous and closed subset of $\left(Q^{*}(X, Y), \tau_{p}\right)$. Then $\mathcal{E}$ is closed also in $\left(F(X, Y), \tau_{p}\right)$.

Proof. The proof follows from Lemma 2.5

Theorem 2.7. Let $X$ be a topological space and $(Y, d)$ be a metric space such that every bounded set is totally bounded. Let $\mathcal{E}$ be a densely equiquasicontinuous subset of $Q^{*}(X, Y)$. Then the topologies $\tau_{p}$ and $\tau_{U C}$ on $\mathcal{E}$ coincide.

Proof. Let $\left\{f_{\lambda}: \lambda \in \Lambda\right\}$ be a net in $\mathcal{E}$ which $\tau_{p}$-converges to $f \in Q^{*}(X, Y)$. We

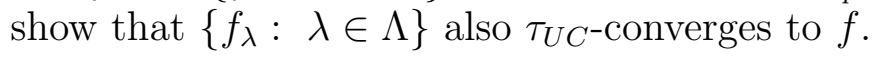

By Lemma 2.4 the set $E \cup\{f\}$ is densely equiquasicontinuous, so for every $x \in X$ and every $m \in \mathbb{N}$ there exists a finite family $\mathcal{B}^{m, x}$ of quasi-open or nowhere dense subsets of $X$ such that $\cup \mathcal{B}^{m, x}$ is a neighbourhood of $x$ and such that for every $g \in \mathcal{E} \cup\{f\}$, for every $B \in \mathcal{B}^{m, x}$ and for every $p, q \in B, d(g(p), g(q))<\frac{1}{m}$.

Let $x \in X, m \in \mathbb{N}$. We claim that there is $\lambda_{m, x} \in \Lambda$ such that $\left.d(f(u)), f_{\lambda}(u)\right) \leq$ $\frac{3}{m}$ for every $\lambda \geq \lambda_{m, x}$ and every $u \in \cup \mathcal{B}^{m, x}$. For every $B \in \mathcal{B}^{m, x}$ we choose a $z \in B$. Since $\left\{f_{\lambda}: \lambda \in \Lambda\right\} \tau_{p}$-converges to $f$, there is $\lambda_{z, B}$ such that $\left.d(f(z)), f_{\lambda}(z)\right)<\frac{1}{m}$ for every $\lambda \geq \lambda_{z, B}$. Let $u \in B, \lambda \geq \lambda_{z, B}$. 


$$
\begin{aligned}
& \left.d\left(f(u), f_{\lambda}(u)\right) \leq d(f(u), f(z))+d(f(z)), f_{\lambda}(u)\right) \leq \\
\leq & \left.\left.d(f(u), f(z))+d(f(z)), f_{\lambda}(z)\right)+d\left(f_{\lambda}(z)\right), f_{\lambda}(u)\right) \leq \\
\leq & \frac{1}{m}+\frac{1}{m}+\frac{1}{m}<\frac{3}{m},
\end{aligned}
$$

Then let $\lambda_{m, x}$ be such that $\lambda_{m, x} \geq \lambda_{z, B}$ for every $B \in \mathcal{B}^{m, x}$. Then we have that $d\left(f(u), f_{\lambda}(u)\right) \leq \frac{3}{m}$ for every $u \in \cup \mathcal{B}^{m, x}$ and every $\lambda \geq \lambda_{m, x}$.

Let $K$ be a compact subset of $X$. For every $m \in \mathbb{N}$ there is finite set of points $x_{j} \in K$, where $j \in\left\{1,2, \cdots, n_{m}\right\}$ such that the family $\cup \mathcal{B}^{m, x_{1}}$, $\cup \mathcal{B}^{m, x_{1}}, \cdots, \cup \mathcal{B}^{m, x_{n_{m}}}$ is a finite cover of $K$. Let $\lambda_{m}$ be such that $\lambda_{m} \geq \lambda_{m, x_{j}}$ for every $j \in\left\{1,2, \cdots, n_{m}\right\}$. Then $\left.d(f(u)), f_{\lambda}(u)\right) \leq \frac{3}{m}$ for every $u \in K$ and every $\lambda \geq \lambda_{m}$. Thus the net $\left\{f_{\lambda}: \lambda \in \Lambda\right\}$ uniformly converges to $f$ on $K$.

Let $Y$ be a metric space. We say that a subset $H$ of $F(X, Y)$ is pointwise bounded provided that for each $x \in X$ the set $H[x]$ is bounded in $Y$.

Let $(Y, d)$ be a metric space. We say that $d$ has the Heine-Borel property if each closed bounded subset is compact. This notion is also known in the literature as the boundedly compact space [2]. Therefore $(Y, d)$ is a locally compact, separable metric space and $d$ is complete. In fact, any locally compact, separable metric space has a compatible metric with the Heine-Borel property ([20], [9]).

Theorem 2.8. Let $X$ be a locally compact space and $(Y, d)$ be a nontrivial metric space such that d has the Heine-Borel property. A subset $\mathcal{E}$ of $\left(Q^{*}(X, Y), \tau_{U C}\right)$ is compact if and only if it is closed, pointwise bounded and densely equiquasicontinuous.

Proof. Let $\mathcal{E}$ be closed in $\left(Q^{*}(X, Y), \tau_{U C}\right)$, pointwise bounded and densely equiquasicontinuous subset of $Q^{*}(X, Y)$. By Theorem $2.7 \mathcal{E}$ is also $\tau_{p}$-closed. Since $\mathcal{E}$ is pointwise bounded for every $x \in X$, from the Heine-Borel property of $d$, there exist a compact set $B_{x}$ such that $\mathcal{E}[x] \subset B_{x}$. The product $\prod_{x \in X}\left\{B_{x}: x \in X\right\}$ is a compact subset of $Y^{X}=\prod_{x \in X}\left\{Y_{x}: x \in X\right\}$ with the relative product topology. Since $\mathcal{E}$ is a $\tau_{p}$-closed subset of the $\tau_{p}$-compact set $\prod_{x \in X}\left\{B_{x}: x \in X\right\}$ it follows that $\mathcal{E}$ is $\tau_{p}$-compact and hence by Theorem $2.7 \mathcal{E}$ is $\tau_{U C^{-}}$-compact.

For the converse, suppose that $\mathcal{E}$ is a compact subset of $\left(Q^{*}(X, Y), \tau_{U C}\right)$. The set $\mathcal{E}$ is closed because $\left(Q^{*}(X, Y), \tau_{U C}\right)$ is a Hausdorff space.

The evaluation at $x$ defined by $e_{x}(f)=f(x)$ for all $f \in Q^{*}(X, Y)$ is continuous with respect to $\tau_{p}$ topology on $Q^{*}(X, Y)$ [15], hence it is continuous also with respect to $\tau_{U C}$ topology on $Q^{*}(X, Y)$ (Theorem 2.7) and so the image $\mathcal{E}[x]$ of $\mathcal{E}$ is compact and therefore is bounded.

For the proof of equiquasicontinuity of $\mathcal{E}$ we use an idea of the proof of Theorem 5.7 in [9]. Let $x \in X$ and let $O$ be an open neighbourhood such that $\bar{O}=A$ is compact. Let $\epsilon>0$, we define a finite family $\mathcal{B}$ of quasi-open subsets of $X$ at follows. Since $\mathcal{E}$ is compact in $\left(Q^{*}(X, Y), \tau_{U C}\right)$, there is $f_{1}, \cdots, f_{n} \in \mathcal{E}$ such that $\mathcal{E} \subset W\left(f_{1}, A, \frac{\epsilon}{3}\right) \cup \cdots \cup W\left(f_{n}, A, \frac{\epsilon}{3}\right)$.

Since every function from $\mathcal{E}$ is locally bounded and $A$ is compact, for every $i \in$ $\{1,2, \cdots, n\}$ the set $f_{i}(A)$ is bounded. Choose $y_{0} \in Y$ and let $r>0$ be 
such that the set $f_{1}(A) \cup \cdots \cup f_{n}(A)$ is contained in the closed ball $B_{r}\left(y_{0}\right)$. Let $V_{1}, V_{2}, \cdots, V_{m}$ be a finite open cover of $B_{r}\left(y_{0}\right)$ where radius of $V_{j}$ is less than $\frac{\epsilon}{3}$ for every $j \in\{1, \cdots, m\}$. For every $i \in\{1,2, \cdots, n\}, j \in\{1,2, \cdots, m\}$ put $B_{j}^{i}=f_{i}^{-1}\left(V_{j}\right)$, which is a quasi-open set since $f_{i}$ is quasicontinuous.

Denote by $\mathcal{F}$ the set of all functions from $\{1,2, \cdots, n\}$ to $\{1,2, \cdots, m\}$. For every $h \in \mathcal{F}$ put

and

$$
P_{h}=\left(O \cap B_{h(1)}^{1} \cap \cdots \cap B_{h(n)}^{n}\right) \backslash \operatorname{Int}\left(O \cap B_{h(1)}^{1} \cap \cdots \cap B_{h(n)}^{n}\right)
$$

$$
R_{h}=\operatorname{Int}\left(O \cap B_{h(1)}^{1} \cap \cdots \cap B_{h(n)}^{n}\right) .
$$

By Remark 2.3 for every $h \in \mathcal{F}$ the set $P_{h}$ is a nowhere dense set.

Denote by $\mathcal{B}$ the family containing all nonempty sets $P_{h}$ and $R_{h}$ where $h \in \mathcal{F}$. We show that $\cup \mathcal{B}$ is a neighbourhood of $x$. Let $z \in O$, then there is $h \in \mathcal{F}$ such that $f_{i}(z) \in V_{h(i)}$ and so $z \in B_{h(i)}^{i}$ for every $i \in\{1,2, \cdots, n\}$. Then $z \in P_{h}$ or $z \in R_{h}$ and thus $z \in \cup \mathcal{B}$.

Now let $f \in \mathcal{E}$, let $B \in \mathcal{B}$ and let $p, q \in B$. Of course there is a $h \in \mathcal{F}$ such that $B \subset B_{h(1)}^{1} \cap \cdots \cap B_{h(n)}^{n}$. Because $\mathcal{E} \subset W\left(f_{1}, A, \frac{\epsilon}{3}\right) \cup \cdots \cup W\left(f_{n}, A, \frac{\epsilon}{3}\right)$ there exist a $i \in\{1,2, \cdots, n\}$ such that $f \in W\left(f_{i}, A, \frac{\epsilon}{3}\right)$. So $d\left(f(p), f_{i}(p)\right)<\frac{\epsilon}{3}$ and $d\left(f(q), f_{i}(q)\right)<\frac{\epsilon}{3}$. Because $p, q \in B_{h(i)}^{i}$ we have that $f_{i}(p) \subset V_{h(i)}$ and $f_{i}(q) \subset V_{h(i)}$ Then $d(f(p), f(q)) \leq d\left(f(p), f_{i}(p)\right)+d\left(f_{i}(p), f_{i}(q)\right)+d\left(f_{i}(q), f(q)\right)<\frac{\epsilon}{3}+\frac{\epsilon}{3}+\frac{\epsilon}{3}=\epsilon$. Hence $\mathcal{E}$ is densely equiquasicontinuous.

\section{Compact subsets of $\left(M U(X, Y), \tau_{U C}\right)$ And $\left(M C(X, Y), \tau_{U C}\right)$}

Let $X, Y$ be Hausdorff topological spaces. By $2^{Y}$ we denote the space of all closed subsets of $Y$ and by $C L(Y)$ the space of all nonempty closed subsets of $Y$. The space of all functions from $X$ to $2^{Y}$ we denote by $F\left(X, 2^{Y}\right)$ and the space of all functions from $X$ to $C L(Y)$ we denote by $F(X, C L(Y))$. We also call the functions from $F\left(X, 2^{Y}\right)$ set-valued maps, or multifunctions, from $X$ to $Y$. If $F$ is a set-valued map from $X$ to $Y$, then its graph is the set $\{(x, y) \in$ $X \times Y: y \in F(x)\}$. Conversely, if $F$ is a subset of $X \times Y$ and $x \in X$, define $F(x)=\{y \in Y:(x, y) \in F\}$. Then we can assign to each subset $F$ of $X \times Y$ a set-valued map which takes the value $F(x)$ at each point $x \in X$ and which graph is $F$. In this way, we identify set-valued maps with their graphs. Following [7] the term map is reserved for a set-valued map.

Given two maps $F, G: X \rightarrow Y$, we write $G \subset F$ and say that $G$ is contained in $F$ if $G(x) \subset F(x)$ for every $x \in X$.

A map $F: X \rightarrow Y$ is upper-semicontinuous at a point $x \in X$ if for every open set $V$ containing $F(x)$, there exists an open set $U \subset X, x \in U$ such that $F(U)=\bigcup\{F(u): u \in U\} \subset V$. F is upper-semicontinuous if it is uppersemicontinuous at each point of $X$.

Following Christensen [6] we say that a map $F$ is usco if it is upper-semicontinuous and takes nonempty compact values. A map $F$ from a topological space $X$ to a linear topological space $Y$ is cusco if it is usco and $F(x)$ is convex for every $x \in X$. 
Finally, a map $F$ from a topological space $X$ to a topological (linear topological space) $Y$ is said to be minimal usco (minimal cusco) if it is a minimal element in the family of all usco (cusco) maps (with domain $X$ and range $Y$ ); that is, if it is usco (cusco) and does not contain properly any other usco (cusco) map from $X$ into $Y$. By an easy application of the Kuratowski-Zorn principle we can guarantee that every usco (cusco) map from $X$ to $Y$ contains a minimal usco (cusco) map from $X$ to $Y$ (see [5], [7]).

We say that a (single-valued) function $f: X \rightarrow Y$ is subcontinuous (see [8]) at $x \in X$ if for every net $\left\{x_{\sigma}: \sigma \in \Sigma\right\}$ in $X$ converging to $x$, there is a convergent subnet of $\left\{f\left(x_{\sigma}\right): \sigma \in \Sigma\right\}$. A function $f$ is subcontinuous if it is subcontinuous at every point of $X$.

Let $F: X \rightarrow Y$ be a map with nonempty values. Then a function $f: X \rightarrow Y$ is called a selection of $F$ if $f(x) \in F(x)$ for every $x \in X$.

Let $Y$ be a Hausdorff locally convex (linear topological) space. Let $F: X \rightarrow Y$ be a map with nonempty compact values. Then a selection $f$ of $F$ such that $f(x)$ is a extreme point of $F(x)$ for every $x \in X$ is called an extreme function of $F$ [11].

In our papers [10], [11] we gave characterizations of minimal usco and minimal cusco maps via their selections.

Theorem 3.1. (see [10]) Let $X, Y$ be topological spaces and $Y$ be a $T_{1}$ regular space. Let $F$ be a map from $X$ to $Y$. The following are equivalent:

(1) $F$ is a minimal usco map;

(2) There exist a quasicontinuous and subcontinuous selection $f$ of $F$ such that $\bar{f}=F$;

(3) Every selection $f$ of $F$ is quasicontinuous, subcontinuous and $\bar{f}=F$.

Theorem 3.2. (see [11]) Let $X$ be a topological space and $Y$ be a Hausdorff locally convex (linear topological) space. Let $F$ be a map from $X$ to $Y$. Then the following are equivalent:

(1) F is a minimal cusco map;

(2) F has nonempty compact values and there is a quasicontinuous, subcontinuous selection $f$ of $F$ such that $\overline{c o} \bar{f}(x)=F(x)$ for every $x \in X$;

(3) F has nonempty compact, convex values, $F$ has a closed graph, every extreme function of $F$ is quasicontinuous, subcontinuous and any two extreme functions of $F$ have the same closures of their graphs;

(4) $F$ has nonempty compact values, every extreme function $f$ of $F$ is quasicontinuous, subcontinuous and $F(x)=\overline{c o} \bar{f}(x)$ for every $x \in X$.

To define a densely continuous form from $X$ to $Y$ [9], denote by $D C(X, Y)$ the set of all functions $f \in F(X, Y)$ such that the set $C(f)$ of points of continuity is dense in $X$. We call such functions densely continuous.

Of course $D C(X, Y)$ contains the set $C(X, Y)$ of all continuous functions from $X$ to $Y$. If $Y=\mathbb{R}$ and $X$ is a Baire space, then all upper and lower semicontinuous functions on $X$ belongs to $D C(X, Y)$ and if $X$ is a Baire space and $Y$ is a metric 
space then every quasicontinuous function $f: X \rightarrow Y$ has a $G_{\delta}$ dense set $C(f)$ of the points of continuity [19]; i.e. $Q(X, Y) \subset D C(X, Y)$.

For every $f \in D C(X, Y)$ we denote by $\overline{f \uparrow C(f)}$ the closure of the graph of $f \uparrow C(f)$. If $D$ is any dense subset of $C(f)$ then the closure of $f \uparrow D$ in $X \times Y$ is equal to $\overline{f \uparrow C(f)}$. We define the set $D(X, Y)$ of densely continuous forms by

$$
D(X, Y)=\{\overline{f\lceil C(f)}: f \in D C(X, Y)\} .
$$

Then the densely continuous forms from $X$ to $Y$ may be considered as maps, where for each $x \in X$ and $F \in D(X, Y) F(x)=\{y \in Y:(x, y) \in F\}$.

Densely continuous forms from $X$ to $Y$ have a kind of minimality property found in the theory of minimal USCO maps. In particular, if $X$ is a Baire space $X$ and $(Y, d)$ is locally compact metric space then $D^{*}(X, Y)=M U(X, Y)$, where $D^{*}(X, Y)$ is the set of all members of $D(X, Y)$ that are locally bounded [14].

We have the following characterizations of elements of $D(X, Y)$.

Theorem 3.3. (see [10]) Let $X$ be a Baire space and $Y$ be a metric space. Let $F: X \rightarrow Y$ be such that $F(x) \neq \emptyset$ for every $x \in X$. The following are equivalent:

(1) $F \in D(X, Y)$

(2) There is a quasicontinuous function $f: X \rightarrow Y$ such that $\bar{f}=F$;

(3) Every selection $f$ of $F$ is quasicontinuous and $\bar{f}=F$.

It is easy to see that if $f \in Q(X, Y)$ and $D$ is a dense subset of $X$ then $\bar{f}=\overline{f \uparrow D}$.

Let $(Y, d)$ be a metric space. The open $d$-ball with center $z_{0} \in Y$ and radius $\epsilon>0$ will be denoted by $S_{\epsilon}\left(z_{0}\right)$ and the $\epsilon$-enlargement $\bigcup_{a \in A} S_{\epsilon}(a)$ for a subset $A$ of $Y$ will be denoted by $S_{\epsilon}(A)$.

If $A \in C L(Y)$, the distance functional $d(., A): Y \mapsto[0, \infty)$ is described by the familiar formula

$$
d(z, A)=\inf \{d(z, a): a \in A\} .
$$

Let $A$ and $B$ be nonempty subsets of $(Y, d)$. The excess of $A$ over $B$ with respect to $d$ is defined by the formula

$$
e_{d}(A, B)=\sup \{d(a, B): a \in A\} .
$$

The Hausdorff (extended-valued) metric $H_{d}$ on $C L(Y)$ [2] is defined by

$$
H_{d}(A, B)=\max \left\{e_{d}(A, B), e_{d}(B, A)\right\} .
$$

We can also use the following equality on $C L(Y)$ :

$$
H_{d}(A, B)=\inf \left\{\epsilon>0: A \subset S_{\epsilon}(B) \text { and } B \subset S_{\epsilon}(A)\right\} .
$$

The topology generated by $H_{d}$ is called the Hausdorff metric topology.

Following [9] we define the topology $\tau_{p}$ of pointwise convergence on $F\left(X, 2^{Y}\right)$. The topology $\tau_{p}$ of pointwise convergence on $F(X, Y)$ is induced by the uniformity $\mathfrak{U}_{p}$ of pointwise convergence which has a base consisting of sets of the form

$$
W(A, \epsilon)=\left\{(\Phi, \Psi): \forall x \in A \quad H_{d}(\Phi(x), \Psi(x))<\epsilon\right\},
$$

where $A$ is from the family of all nonempty finite subsets of $X$ and $\epsilon>0$. 
We will define the topology $\tau_{U C}$ of uniform convergence on compact sets on $F\left(X, 2^{Y}\right)$ [9]. This topology is induced by the uniformity $\mathfrak{U}_{U C}$ which has a base consisting of sets of the form

$$
W(K, \epsilon)=\left\{(\Phi, \Psi): \forall x \in K \quad H_{d}(\Phi(x), \Psi(x))<\epsilon\right\},
$$

where $K$ is from the family of all nonempty compact subsets of $X$ and $\epsilon>0$.

Theorem 3.4. Let $X$ be a locally compact space and $(Y, d)$ be a nontrivial metric space such that $d$ has the Heine-Borel property. If $\mathcal{E}$ is a compact subset of $\left(Q(X, Y), \tau_{U C}\right)$ then $\{\bar{f}: f \in \mathcal{E}\}$ is a compact subset of $\left(D(X, Y), \tau_{U C}\right)$.

Proof. Let $\mathcal{E}$ be a compact subset of $\left(Q(X, Y), \tau_{U C}\right)$ and let $\left\{\bar{f}_{\lambda}: \lambda \in \Lambda\right\}$ be a net in $\{\bar{f}: f \in \mathcal{E}\}$. By Theorem 3.3 if $f \in \mathcal{E}$, then $\bar{f} \in D(X, Y)$. By passing to a subnet, there is a function $f \in \mathcal{E}$ such that the net $\left\{f_{\lambda}: \lambda \in \Lambda\right\} \tau_{U C}$-converges to $f$. We prove that $\left\{\bar{f}_{\lambda}: \lambda \in \Lambda\right\} \tau_{U C^{-}}$-converges to $\bar{f}$. For every $x \in X$ choose a compact set $K_{x} \subset X$, such that $x \in \operatorname{Int} K_{x}$. Let $x_{0} \in X$. First we show that $\left\{\bar{f}_{\lambda}: \lambda \in \Lambda\right\}$ uniformly converges on $\operatorname{Int} K_{x_{0}}$ to $\bar{f}$.

Let $\epsilon>0$. The net $\left\{f_{\lambda}: \lambda \in \Lambda\right\}$ uniformly converges to $f$ on $K_{x_{0}}$, so there is $\lambda_{0} \in \Lambda$ such that $d\left(f(x), f_{\lambda}(x)\right)<\frac{\epsilon}{2}$ for every $\lambda \geq \lambda_{0}$ and every $x \in K_{x_{0}}$.

Choose any $x \in \operatorname{Int} K_{x_{0}}$ and $z \in \bar{f}(x)$. There is a net $\left\{x_{\omega}: \omega \in \Omega\right\}$ in $\operatorname{Int} K_{x_{0}}$ which converges to $x$ such that the net $\left\{f\left(x_{\omega}\right): \omega \in \Omega\right\}$ converges in $Y$ to $z$. Without loss of generality we may assume that $\left\{f\left(x_{\omega}\right): \omega \in \Omega\right\}$ is bounded. Let $\lambda$ be arbitrary such that $\lambda \geq \lambda_{0}$. Then $d\left(f\left(x_{\omega}\right), f_{\lambda}\left(x_{\omega}\right)\right)<\frac{\epsilon}{2}$ for every $\omega \in \Omega$. The net $\left\{f_{\lambda}\left(x_{\omega}\right): \omega \in \Omega\right\}$ has a cluster point $y \in \bar{f}_{\lambda}(x)$ since is bounded and $d$ has the Heine-Borel property. Then $d(z . y) \leq \frac{\epsilon}{2}$ and since $z$ is arbitrary element of $\bar{f}(x)$ we have that $\bar{f}(x) \subset S_{\epsilon}\left(\bar{f}_{\lambda}(x)\right)$. The inclusion $\bar{f}_{\lambda}(x) \subset S_{\epsilon}(\bar{f}(x))$ can be proved similarly, hence $H_{d}\left(\bar{f}(x), \bar{f}_{\lambda}(x)\right)<\epsilon$. This shows that for every $x \in \operatorname{Int} K_{x_{0}}$ and every $\lambda \geq \lambda_{0} H_{d}\left(\bar{f}(x), \bar{f}_{\lambda}(x)\right)<\epsilon$. So the net $\left\{\bar{f}_{\lambda}: \lambda \in \Lambda\right\}$ uniformly converges on $\operatorname{Int} K_{x_{0}}$ to $\bar{f}$.

Let $K$ be a compact subset of $X$. There are finitely many points $x_{1}, x_{2}, \cdots, x_{n}$ from $X$ such that $K \subset \cup\left\{\right.$ Int $\left.K_{x_{i}}: i \in\{1,2, \cdots, n\}\right\}$. The uniform convergence of $\left\{\bar{f}_{\lambda}: \lambda \in \Lambda\right\}$ to $\bar{f}$ on $K$ follows from uniform convergence of this net to $\bar{f}$ on Int $_{x_{i}}$ for $i \in\{1,2, \cdots, n\}$.

Denote by $M U(X, Y)$ the set of all minimal usco maps from $X$ to $Y$ and if $Y$ is a Hausdorff locally convex linear topological space, by $M C(X, Y)$ the set of all minimal cusco maps from $X$ to $Y$.

If $F$ is a map from $X$ to a metric space $(Y, d)$, we say that $F$ is locally bounded, if for every $x \in X$ there is an open set $U \subset X, x \in U$ such that $F(U)=\bigcup\{F(u)$ : $u \in U\}$ is a bounded subset of $(Y, d)$.

Note that if $f$ is a selection of $F \in M U(X, Y)$ then $f \in Q(X, Y)$ (Theorem 3.1) and since $F$ is upper-semicontinuous, $f \in Q^{*}(X, Y)$.

Theorem 3.5. Let $X$ be a locally compact space and $(Y, d)$ be a nontrivial metric space such that $d$ has the Heine-Borel property. Let $\mathcal{E}$ be a subset of $M U(X, Y)$, where for every $F \in \mathcal{E}$ there is a selection $f_{F}$ of $F$ such that $\left\{f_{F}: F \in \mathcal{E}\right\}$ is closed in $\left(Q^{*}(X, Y), \tau_{U C}\right)$, densely equiquasicontinuous and pointwise bounded. Then $\mathcal{E}$ is compact subset of $\left(M U(X, Y), \tau_{U C}\right)$. 
Proof. Let the set $\left\{f_{F}: F \in \mathcal{E}\right\}$ be closed in $\left(Q^{*}(X, Y), \tau_{U C}\right)$, densely equiquasicontinuous and pointwise bounded. Then by Theorem 2.8 the set $\left\{f_{F}: F \in \mathcal{E}\right\}$ is a compact subset of $\left(Q^{*}(X, Y), \tau_{U C}\right)$ and by Theorem $3.4\left\{\overline{f_{F}}: F \in \mathcal{E}\right\}$ is a compact subset of $\left(D(X, Y), \tau_{U C}\right)$. By Theorem 3.1 $\mathcal{E}=\left\{\overline{f_{F}}: F \in \mathcal{E}\right\}$, hence $\mathcal{E}$ is a compact subset of $\left(M U(X, Y), \tau_{U C}\right)$.

Let $Y$ be a Hausdorff locally convex (linear topological) space. Define the function $\varphi: M U(X, Y) \rightarrow F(X, C L(Y))$ as follows: $\varphi(F)(x)=\overline{c o} F(x)$.

Theorem 3.6. (see [12]) Let $X$ be a locally compact space and $Y$ be a Banach space. The map $\varphi$ from $\left(M U(X, Y), \tau_{U C}\right)$ onto $\left(M C(X, Y), \tau_{U C}\right)$ is homeomorphism.

Remark 3.7. Let $X$ be a topological space and $Y$ be a Hausdorff locally convex (linear topological) space in which the closed convex hull of a compact set is compact. Note that if $G \in M C(X, Y)$ and $F \in M U(X, Y)$, where $F$ is contained in $G$ then $\varphi(F)(x)=G(x)$ (see [12]). In fact, By Theorem 2.5. in [12] for $G$ there is a unique minimal usco map $F_{G}$ contained in $G$, so $F_{G}=F$. By Lemma 2.1 (see [12]) the map $x \rightarrow \overline{c o} F(x)$ is a cusco map such that $\overline{c o} F(x) \subset G(x)$ for every $x \in X$. Since $G$ is minimal cusco, $G(x)=\overline{c o} F(x)$ for every $x \in X$.

Theorem 3.8. Let $X$ be a locally compact space. Let $\mathcal{E}$ be a subset of $M C\left(X, \mathbb{R}^{n}\right)$ where for every $F \in \mathcal{E}$ there is a selection $f_{F}$ of $F$ such that $\left\{f_{F}: F \in \mathcal{E}\right\}$ is closed in $\left(Q^{*}\left(X, \mathbb{R}^{n}\right), \tau_{U C}\right)$, densely equiquasicontinuous and pointwise bounded. Then $\mathcal{E}$ is a compact subset of $\left(M C\left(X, \mathbb{R}^{n}\right), \tau_{U C}\right)$.

Proof. Let the set $\left\{f_{F}: F \in \mathcal{E}\right\}$ be closed in $\left(Q^{*}\left(X, \mathbb{R}^{n}\right), \tau_{U C}\right)$, densely equiquasicontinuous and pointwise bounded. Then by Theorem 2.8 the set $\left\{f_{F}: F \in \mathcal{E}\right\}$ is a compact subset of $\left(Q^{*}\left(X, \mathbb{R}^{n}\right), \tau_{U C}\right)$ and by Theorem $3.4\left\{\overline{f_{F}}: F \in \mathcal{E}\right\}$ is compact subset of $\left(D\left(X, \mathbb{R}^{n}\right), \tau_{U C}\right)$. By Theorem $3.1\left\{\overline{f_{F}}: F \in \mathcal{E}\right\} \subset M U\left(X, \mathbb{R}^{n}\right)$ and hence $\left\{\overline{f_{F}}: F \in \mathcal{E}\right\}$ is a compact subset of $\left(M U(X, Y), \tau_{U C}\right)$. By Remark $3.7 \mathcal{E}=\varphi\left(\left\{\overline{f_{F}}: F \in \mathcal{E}\right\}\right)$ and by Theorem $3.6 \mathcal{E}$ is a compact subset of $\left(M C\left(X, \mathbb{R}^{n}\right), \tau_{U C}\right)$.

Let $F: X \rightarrow \mathbb{R}$ be a map with bounded values. Define the functions $s^{F}$ and $l_{F}$ as follows:

$$
s^{F}(x)=\sup \{y: y \in F(x)\}, \quad l_{F}(x)=\inf \{y: y \in F(x)\} .
$$

If $F \in M U(X, \mathbb{R}) \cup M C(X, \mathbb{R})$, then of course $s^{F}$ and $l_{F}$ are selections of $F$, where $s^{F}$ is upper-semicontinuous, $l_{F}$ is lower-semicontinuous and both of them are locally bounded. If $F \in M U(X, \mathbb{R})$, by Theorem $3.1, s^{F}$ and $l_{F}$ are quasicontinuous and if $F \in M C(X, \mathbb{R})$ then $s^{F}$ and $l_{F}$ are extreme functions and so by Theorem 3.2 are also quasicontinuous.

Denote by $U C(X, \mathbb{R})$ the set of all upper-semicontinuous functions from $X$ to $\mathbb{R}$.

Define the mapping $\mathcal{S}: M U(X, \mathbb{R}) \cup M C(X, \mathbb{R}) \rightarrow Q^{\star}(X, \mathbb{R}) \cap U C(X, \mathbb{R})$ by $\mathcal{S}(F)=s^{F}$. 
Theorem 3.9. (see [10]) Let $X$ be a locally compact topological space. Then the mapping $\mathcal{S}$ from $\left(M U(X, \mathbb{R}), \mathfrak{U}_{U C}\right)$ onto $\left(Q^{\star}(X, \mathbb{R}) \cap U C(X, \mathbb{R}), \mathfrak{U}_{U C}\right)$ is a uniform isomorphism.

Proposition 3.10. Let $X$ be a Hausdorff topological space. The mapping $\mathcal{S}$ : $M C(X, \mathbb{R}) \rightarrow Q^{\star}(X, \mathbb{R}) \cap U C(X, \mathbb{R})$ is a bijection.

Proof. The map $\varphi^{-1}$ from $M C(X, \mathbb{R})$ to $M U(X, \mathbb{R})$ and map $\mathcal{S}$ from $M U(X, \mathbb{R})$ to $Q^{\star}(X, \mathbb{R}) \cap U C(X, \mathbb{R})$ are bijections. Then also the composition $\mathcal{S} \varphi^{-1}$ from $M C(X, \mathbb{R})$ to $Q^{\star}(X, \mathbb{R}) \cap U C(X, \mathbb{R})$ is a bijection. Let $F \in M U(X, \mathbb{R})$ and let $G(x)=\overline{c o} F(x)$ for every $x \in X$. Clearly $s^{F}=s^{G}$. Then for every $G \in M C(X, \mathbb{R})$ we have that $\mathcal{S}\left(\varphi^{-1}(G)\right)=\mathcal{S}(F)=s^{F}=s^{G}=\mathcal{S}(G)$.

Remark 3.11. It is easy to see that if $A$ and $B$ are nonempty compact subsets of $\mathbb{R}$, then $d(\sup A, \sup B) \leqq H_{d}(A, B)$.

Theorem 3.12. Let $X$ be a locally compact topological space. Then the mapping $\mathcal{S}$ from $\left(M C(X, \mathbb{R}), \mathfrak{U}_{U C}\right)$ onto $\left(Q^{\star}(X, \mathbb{R}) \cap U C(X, \mathbb{R}), \mathfrak{U}_{U C}\right)$ is a uniform isomorphism.

Proof. As we proved above the mapping $\mathcal{S}$ from $M C(X, \mathbb{R})$ to $Q^{\star}(X, \mathbb{R}) \cap U C(X, \mathbb{R})$ is a bijection. By Remark 3.11 we have that $\mathcal{S}:\left(M C(X, \mathbb{R}), \mathfrak{U}_{U C}\right) \rightarrow\left(O^{\star}(X, \mathbb{R}) \cap\right.$ $\left.U C(X, \mathbb{R}), \mathfrak{U}_{U C}\right)$ is uniformly continuous.

To prove that also $\mathcal{S}^{-1}$ is uniformly continuous, let $K$ be a nonempty compact subset of $X$ and $\varepsilon>0$. The local compactness of $X$ implies that there is an open set $O$ in $X$ such that $K \subset O$ and $\bar{O}$ is compact. Let $f, g \in Q^{\star}(X) \cap U C(X)$ be such that $d(f(x), g(x))<\varepsilon$ for every $x \in \bar{O}$. We prove that $H_{d}(\operatorname{co} \bar{f}(x), \operatorname{co} \bar{g}(x)) \leq \varepsilon$ for every $x \in K$. Suppose, by the way of contradiction that this is not true. Then there exist $x_{0} \in K$ such that $H_{d}\left(\operatorname{co} \bar{f}\left(x_{0}\right), \operatorname{co} \bar{g}\left(x_{0}\right)\right)>\epsilon$.

Since $f(x)=\sup c o \bar{f}(x)), g(x)=\sup \operatorname{co} \bar{g}(x))$ and $d(f(x), g(x))<\varepsilon$ for every $x \in X$ we have that $d\left(\inf \operatorname{co} \bar{f}\left(x_{0}\right)\right.$, inf $\left.\operatorname{cog}\left(x_{0}\right)\right)>\epsilon$, where one of following two cases can occurs: $\inf \operatorname{co} \bar{f}\left(x_{0}\right)<\inf \operatorname{co} \bar{g}\left(x_{0}\right)$ or $\inf \operatorname{co} \bar{g}\left(x_{0}\right)<\inf \operatorname{co} \bar{f}\left(x_{0}\right)$. Suppose the first case occurs; the proof for the other one is analogous.

Put $\beta=d\left(\inf \operatorname{co} \bar{f}\left(x_{0}\right)\right.$, inf $\left.\operatorname{co} \bar{g}\left(x_{0}\right)\right)-\epsilon$. Let $\left\{x_{\lambda}: \lambda \in \Lambda\right\}$ be a net in $X$ converging to $x_{0}$, such that the net $\left\{f\left(x_{\lambda}\right): \lambda \in \Lambda\right\}$ converges to inf $\operatorname{co} \bar{f}\left(x_{0}\right)$. Then for $\frac{\beta}{4}$ there is $\lambda_{0} \in \Lambda$ such that $f\left(x_{\lambda}\right) \in S_{\frac{\beta}{4}}\left(\inf \operatorname{co} \bar{f}\left(x_{0}\right)\right)$ for all $\lambda>\lambda_{0}$. The upper semicontinuity of $\operatorname{cog}(x)$ at $x_{0}$ implies that there is an open neighbourhood $U$ of $x_{0}$ such that $\operatorname{cog}(x) \subset S_{\frac{\beta}{4}}\left(\operatorname{cog}\left(x_{0}\right)\right)$ for all $x \in U$. Let $\lambda \in \Lambda$ be such that $\lambda>\lambda_{0}$ and $x_{\lambda} \in U \cap O$. Then of course $d\left(f\left(x_{\lambda}\right), g\left(x_{\lambda}\right)\right)>\epsilon$, a contradiction.

We can give a similar result for functions $l_{F}$, where $F \in M U(X, \mathbb{R})$ or $F \in$ $M C(X, \mathbb{R})$.

Theorem 3.13. Let $X$ be a locally compact space. Let $\mathcal{E}$ be a subset of $\left(M U(X, \mathbb{R}), \tau_{U C}\right)$. Then $\mathcal{E}$ is compact if and only if the set $\left\{s^{F}: F \in \mathcal{E}\right\}$ is closed in $\left(Q^{*}(X, \mathbb{R}), \tau_{U C}\right)$, densely equiquasicontinuous and pointwise bounded.

Proof. Let $\mathcal{E}$ be a compact subset of $\left(M U(X, \mathbb{R}), \tau_{U C}\right)$. By Theorem 3.9 the set $\left\{s^{F}: F \in \mathcal{E}\right\}$ is compact subset of $\left(Q^{*}(X, Y), \tau_{U C}\right)$ and by Theorem 2.8 it is closed in $\left(Q^{*}(X, \mathbb{R}), \tau_{U C}\right)$, densely equiquasicontinuous and pointwise bounded. 
The converse follows from Theorem 3.5.

Theorem 3.14. Let $X$ be a locally compact space. Let $\mathcal{E}$ be a subset of $\left(M C(X, \mathbb{R}), \tau_{U C}\right)$. Then $\mathcal{E}$ is compact if and only if the set $\left\{s^{F}: F \in \mathcal{E}\right\}$ is closed in $\left(Q^{*}(X, \mathbb{R}), \tau_{U C}\right)$, densely equiquasicontinuous and pointwise bounded.

Proof. Let $\mathcal{E}$ be a compact subset of $\left(M C(X, \mathbb{R}), \tau_{U C}\right)$. By Theorem 3.12 the set $\left\{s^{F}: F \in \mathcal{E}\right\}$ is compact subset of $\left(Q^{*}(X, Y), \tau_{U C}\right)$ and by Theorem 2.8 it is closed in $\left(Q^{*}(X, \mathbb{R}), \tau_{U C}\right)$, densely equiquasicontinuous and pointwise bounded.

The converse follows from Theorem 3.8.

Theorem 3.15. Let $X$ be a locally compact space. Let $\mathcal{E}$ be a subset of $\left(M U(X, \mathbb{R}), \tau_{U C}\right)$ such that the set $\left\{s^{F}: F \in \mathcal{E}\right\}$ is densely equiquasicontinuous subset of $Q^{*}(X, \mathbb{R})$. Then the topologies $\tau_{p}$ and $\tau_{U C}$ on $\mathcal{E}$ coincide.

Proof. Let $\left\{F_{\lambda}: \lambda \in \Lambda\right\}$ be a net in $\mathcal{E}$ which $\tau_{p}$-converges to a $F \in \mathcal{E}$. We show

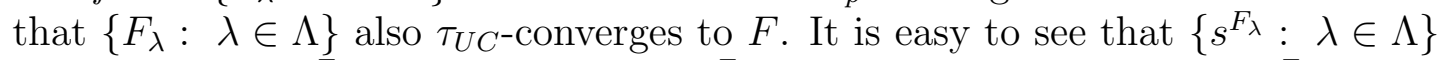
$\tau_{p}$-converges to $s^{F}$. By Theorem $2.7\left\{s^{F_{\lambda}}: \lambda \in \Lambda\right\} \tau_{U C^{-}}$-converges to $s^{F}$ and by Theorem $3.9\left\{F_{\lambda}: \lambda \in \Lambda\right\} \tau_{U C}$-converges to $F$.

Theorem 3.16. Let $X$ be a locally compact space. Let $\mathcal{E}$ be a subset of $\left(M C(X, \mathbb{R}), \tau_{U C}\right)$ such that the set $\left\{s^{F}: F \in \mathcal{E}\right\}$ is densely equiquasicontinuous subset of $Q^{*}(X, \mathbb{R})$. Then the topologies $\tau_{p}$ and $\tau_{U C}$ on $\mathcal{E}$ coincide.

Proof. The proof is similar to the proof of the above Theorem but we must use Theorem 3.12 instead of Theorem 3.9.

Acknowledgement. Author would like to thank to grant APVV-0269-11.

\section{REFERENCES}

1. R. Baire, Sur les functions des variables reelles, Ann. Mat. Pura Appl. 3 (1899), 1-122.

2. G. Beer, Topologies on closed and closed convex sets, Kluwer Academic Publishers, 1993.

3. J.M. Borwein and W.B. Moors, Essentially smooth Lipschitz functions, J. Funct. Anal. 149 (1997), 305-351.

4. J. Borsík, Quasiuniform limits of quasicontinuous functions, Math. Slovaca 42 (1992), 269274.

5. J.M. Borwein and Q.J. Zhu, Multifunctional and functional analytic techniques in nonsmooth analysis, in Nonlinear Analysis, Differential Equations and Control, F.H. Clarke and R.J. Stern, editors, Kluwer Academic Publishers, 1999.

6. J.P.R. Christensen, Theorems of Namioka and R.E. Johnson type for upper semicontinuous and compact valued mappings, Proc. Amer. Math. Soc. 86 (1982), 649-655.

7. L. Drewnowski and I. Labuda, On minimal upper semicontinuous compact valued maps, Rocky Mountain J. Math.20 (1990), 737-752.

8. R.V. Fuller, Sets of points of discontinuity, Proc. Amer. Math. Soc. 38 (1973), 193-197.

9. S.T. Hammer and R.A. McCoy Spaces of densely continuous forms, Set-Valued Anal. 5 (1997), 247-266.

10. Ľ. Holá and D. Holý Minimal usco maps, densely continuous forms and upper semicontinuous functions, Rocky Mountain J. Math. 39 (2009), 545-562.

11. Ľ. Holá and D. Holý New characterization of minimal CUSCO maps, Rocky Mountain J. Math. (to appear).

12. Ľ. Holá and D. Holý Relation betwen minimal USCO and minimal CUSCO maps Port. Math. 70 (2013), Fracs 3, 211-224. 
13. Ľ. Holá Spaces of Densely Continuous Forms, USCO and Minimal USCO Maps, Set-Valued Anal. 11 (2003), 133-151.

14. D. Holý and P. Vadovič Hausdorff graph topology, proximal graph topology and the uniform topology for densely continuous forms and minimal USCO maps, Acta Math. Hungarica 116, (2007), no. 1-2, 133-144.

15. J.L. Kelley General Topology, Van Nostrand, New York, 1955.

16. S. Kempisty Sur les fonctions quasi-continues, 19 (1932), 184-197.

17. M. Matejdes Minimality of multifunctions, Real Anal. Exchange 32 (2007), 519-526.

18. R.A. McCoy Spaces of semicontinuous forms, Topology Proc. 23 (1998), 249-275.

19. T. Neubrunn Quasi-continuity, Real Anal. Exchange 14 (1988), 259-306.

20. H. VaughanOn locally compact metrizable spaces, Bull. Amer. Math. Soc. 43 (1937), 532535 .

Department of Mathematics and Computer Science, Faculty of Education, TRNAVA UNIVERSITY,

Priemyselná 4, 91843 Trnava, Slovakia.

E-mail address: dusan.holy@truni.sk 